\section{Cureus}

\title{
A Systematic Review of Autohemotherapy as a Treatment for Urticaria and Eczema
}

\author{
Devon D. Brewer ${ }^{1}$ \\ 1. Interdisciplinary Scientific Research, Seattle, WA
}

$\square$ Corresponding author: Devon D. Brewer, dbrewer@interscientific.net

Disclosures can be found in Additional Information at the end of the article

\section{Abstract}

The injection of autologous whole blood or serum, known as autohemotherapy, was a standard dermatologic treatment in the early 1900s. Conventional dermatologists eventually abandoned autohemotherapy due to a lack of supporting evidence, even though there had been no formal attempts to assess its effectiveness. Recently, several investigators have evaluated autohemotherapy as a treatment for urticaria and eczema. I conducted a systematic review of the literature on autohemotherapy, focusing on treatment outcomes. The available evidence indicates that autohemotherapy does not have major side effects, and that minor adverse effects are short-lived and similar in frequency to those from placebo injections. Overall, autohemotherapy tends to be somewhat more effective in reducing symptoms than control therapy across studies, although the advantage is not statistically reliable. Urticaria patients who test positive on the autologous serum skin test display a moderately better response to autohemotherapy than patients who test negative. Based on the limited evidence available, autologous whole blood and autologous serum injections appear to have similar effectiveness. Furthermore, the severity of symptoms prior to treatment is not consistently related to patients' apparent response to autohemotherapy.

Categories: Dermatology, Allergy/Immunology

Keywords: meta-analysis, dermatology, autohemotherapy, urticaria, eczema

\section{Introduction}

Autohemotherapy involves injecting autologous whole blood or autologous serum, typically into muscle. In 1913, Ravaut [1] and Spiethoff [2] described their use of autohemotherapy for various dermatologic conditions. Soon thereafter, autohemotherapy became a standard treatment for many dermatologic disorders, including urticaria and eczema, in Europe, North America, and Japan [3-5].

Received 11/23/2014

Review began 11/23/2014

Review ended 12/05/2014

Published 12/09/2014

C) Copyright 2014

Brewer. This is an open access article distributed under the terms of the Creative Commons Attribution License CC-BY 3.0., which permits unrestricted use, distribution, and reproduction in any medium, provided the original author and source are credited.
After several decades, however, autohemotherapy fell into disrepute because of a lack of rigorous supporting evidence [6]. There also seems to have been no attempt at the time to appraise the therapy systematically. In more recent decades, autohemotherapy has been used to treat dermatologic and other diseases, mainly by alternative medical providers, especially in Europe [7] and Latin America.

In the last 15 years, conventional dermatologists re-discovered autohemotherapy and submitted it to rigorous evaluation. When these studies began, the autologous serum skin test (ASST) [8-9] was increasingly employed as a tool for diagnosing auto-reactive urticaria in clinical care and research. For this test, a small amount of the patient's blood is drawn and spun, with the serum extracted and then re-injected intradermally into the patient on a clear 
patch of skin, next to sites where negative (saline) and positive (histamine) controls have been injected. If the wheal response to the autologous serum is substantially greater $(\geqslant 1.5 \mathrm{~mm})$ than that to the negative control, the result is interpreted as positive, or is otherwise considered negative. Because autohemotherapy had long been thought to ameliorate dermatologic conditions presumed to have an auto-reactive component, many of those studying autohemotherapy assessed whether urticaria patients' response to autohemotherapy differed by ASST status.

In this systematic review, I synthesize the evidence on autohemotherapy as a treatment for chronic spontaneous/idiopathic urticaria and eczema. I examine five aspects of autohemotherapy: 1) adverse effects, 2) overall effectiveness in reducing symptoms, 3) effectiveness by ASST status, 4) relative effectiveness of autologous whole blood injections and autologous serum injections, and 5) effectiveness in relation to symptom severity before treatment.

\section{Materials And Methods}

Literature Search, Retrieval, and Inclusion Criteria

To identify potentially relevant reports, I searched Scopus (www.scopus.com), which covers all journals included in PubMed and EMBASE as well as other sources, and Google Scholar (scholar.google.com) with the following search terms: (autohemotherapy OR autohaemotherapy OR ("autologous whole blood" AND inject*) OR ("autologous serum" AND inject*)) AND (urticaria OR angioedema OR eczema OR dermatitis OR xerosis).

I also used an abbreviated set of search terms (autohemotherapy OR autohaemotherapy OR "autologous whole blood" OR "autologous serum") to search the following additional databases for reports or leads to reports:

Health Services Research Projects in Progress

- National Digital Library of Theses and Dissertations (international)

- DART-Europe E-theses Portal

DiVA (Scandinavian theses and dissertations)

- National Electronic Theses and Dissertations Portal (South Africa)

EThOS (Electronic Theses Online Service) [UK]

- ProQuest Dissertations and Theses (international)

Theses Canada

ProQuest COS Conference Paper Index

- OpenGrey (Europe)

F1000 Posters

Figshare

online abstracts for conferences held by the American Academy of Allergy, Asthma, and Immunology, American Academy of Dermatology, Canadian Dermatology Association, European Academy of Allergy and Clinical Immunology, Medical Dermatology Society, and Society for Investigative Dermatology (other relevant major professional societies do not appear to have searchable online archives of meeting abstracts or their meeting abstracts are captured in other databases I searched)

- Harvard Dataverse Network

- International Clinical Trials Registry Platform

- National Institutes of Health RePORTER (grant database, USA)

- UK Clinical Research Network Study Portfolio

In addition, I examined my personal library, references in reports retrieved from these searches, 
and reports citing reports included in the review (as indicated by Google Scholar) for relevant reports. I conducted the literature search in August of 2014.

I sought full reports for those abstracts that described clinical trials, case studies/series, and other observational studies involving the clinical evaluation of autohemotherapy for urticaria or eczema in patients over one year old. Although I conducted the search in English, I considered all reports identified, regardless of language. I retrieved screened reports from free/open access sources online, through the electronic and physical holdings of the University of Washington libraries, and directly from authors themselves. If I was unable to obtain a full report through these means, I relied on the information reported in the abstract.

In the review, I included only those reports with study designs and results corresponding to one or more of the clinical aspects on which I focused (see the next section for more details). I inspected reports that did not meet these inclusion criteria for additional relevant references. In some reports, the authors did not describe one or more potentially relevant results sufficiently to be included in the review. In such cases, I contacted the corresponding authors of such studies by email (at least two attempts) or post (one attempt) to request the data or ask the authors to perform the particular analyses and share the results. For reports written in languages other than English, I used Google Translate (translate.google.com) to assist with translation. When numeric values necessary for analysis were not reported precisely in an article and authors did not respond to my requests for such information, I estimated values manually from graphical displays in the articles (if possible).

Analysis

In this review, the primary outcome is symptom activity or severity as assessed at the end of a study's follow-up period. This outcome is the most clinically relevant. Focusing on the end of follow-up, after a period of no treatment, may also reduce the influence of placebo effects. In studies where end of follow-up measures are not available, I relied on end of treatment assessments and summarized them separately.

To assess the overall effectiveness of autohemotherapy, I included randomized controlled trials with designs that allow the independent effect of autohemotherapy on symptom activity or severity to be determined. I summarized results based on different types of disease (urticaria vs. eczema), controls (placebo vs. no placebo), and outcome measures (end of follow-up scores vs. proportional change from baseline to end of follow-up) separately.

I focused on two types of results to assess the differential effectiveness of autohemotherapy by patient ASST status. One type comes from randomized controlled trials in which the data for ASST+ and ASST- urticaria patients can be analyzed separately. The second type of results are direct comparisons of outcomes for ASST+ and ASST- patients who received autohemotherapy.

To evaluate the relative effectiveness of autologous whole blood injections and autologous serum injections, I relied on randomized trials in which these two variants of autohemotherapy were compared head-to-head.

To examine the relationship between symptom severity at baseline and response to autohemotherapy, I included reports that included individual level data on symptom severity at baseline and the end of follow-up.

I conducted one set of analyses that was not planned before the beginning of data analysis. These analyses focused on the response to placebo injections by ASST status and paralleled those on the differential response to autohemotherapy by ASST status. 
The main effect size measure I used is the response ratio [10-11]. The response ratio is the ratio of the mean of one group to the mean of another group. In this review, these comparisons were between autohemotherapy and control, ASST+ patients and ASST- patients, and autologous whole blood injections and autologous serum injections. To measure the association between baseline severity of symptoms and response to autohemotherapy, I calculated Pearson correlations.

I computed random-effects summary (pooled) estimates of effect sizes in which the included effect sizes were weighted by their precision (inverse variance or square of the standard error) [11-13]. To measure the heterogeneity of included effect sizes, I calculated the $\mathrm{I}^{2}$ statistic [14] which ranges between 0 and 1 . $\mathrm{I}^{2}$ indicates the proportion of observed variance in effect sizes beyond that from sampling error in the individual studies summarized. It thus serves as a measure of inconsistency of results across studies, relative to the studies' precision. I also computed the corresponding $95 \%$ confidence intervals for effect sizes from individual reports and the summaries. In studies with small sample sizes (or summaries based on them), the confidence interval for the response ratio can be asymmetric [11]. In computing summaries for the correlation coefficients, I used Fisher's z transformations [11]. I display results for individual and summary effect sizes (and their confidence intervals) with forest plots. I performed all analyses with Gnumeric Spreadsheet (version 1.10.17; www.gnumeric.org).

The protocol and all results for this systematic review are archived publicly at: https://osf.io/gzc3b/?view_only=efd7067aef3a44ad825d212a62702bc5. Except for analyses comparing outcomes in urticaria placebo patients by ASST status, all analyses I describe in this article were planned before analysis began, and mostly before the literature search began.

\section{Results}

\section{Literature Search and Eligible Reports}

Figure 1 summarizes the results of my literature search and assessment of the reports I found. Given the diversity of databases I searched and the lack of standardized bibliographic formats in these databases, I did not attempt to ascertain the number of unduplicated reports.

Nonetheless, the overlap appeared modest, with the greatest absolute overlap between Scopus and Google Scholar.

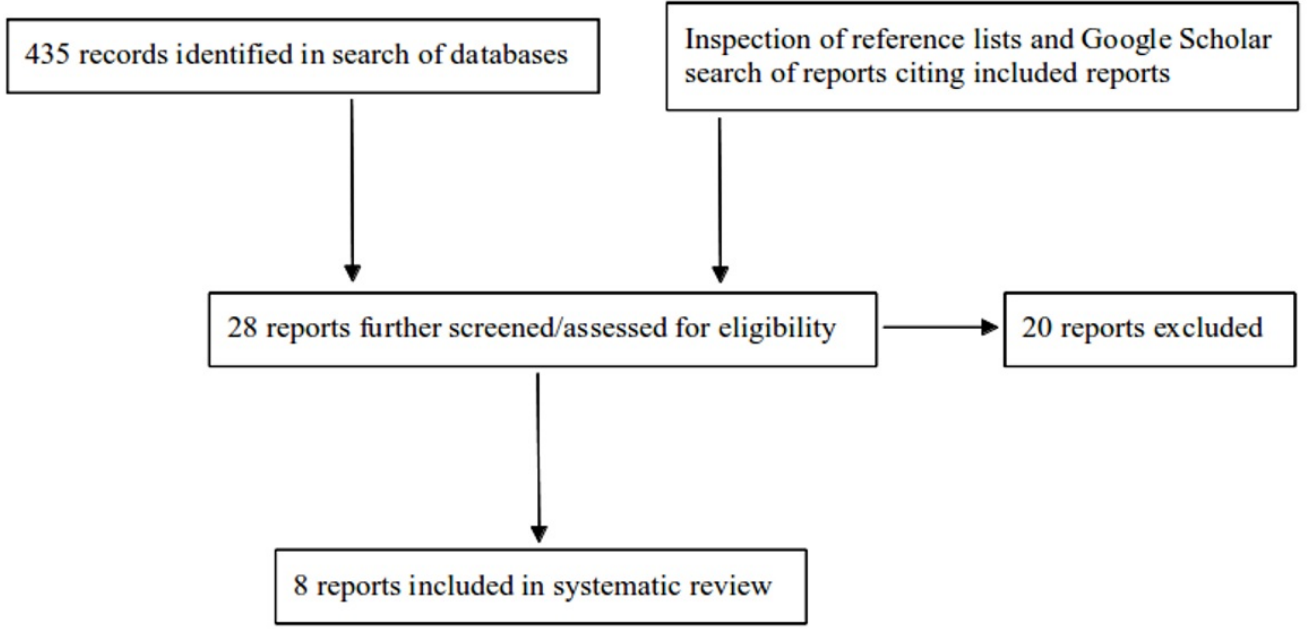

FIGURE 1: Summary of results of literature search and
assessment of reports 


\section{Cureus}

I included eight reports in the systematic review. Table 1 shows the reasons for excluding 20 other reports. Among the excluded studies, there were four randomized controlled trials (no placebo) with designs that seemingly would allow the independent effects of autohemotherapy to be assessed [15-18]. The abstracts of these trials indicated the results favored autohemotherapy. However, I could not compute effect sizes for these trials because the results reported in the abstracts were either incomplete or internally inconsistent, and the authors did not respond to my postal requests for their articles. 


\section{Cureus}

\section{Study ${ }^{1} \quad$ Reason for Exclusion}

Hiraizumi

No results related to research questions

Chopra [43]

Apparently non-randomized comparison between autohemotherapy and "other modalities", no quantitative results

Mori [4] No results related to research questions

Yang [44] Incomplete or internally inconsistent results in abstract; full article not available

Liang [15] Incomplete or internally inconsistent results in abstract; full article not available

Min [45] Design does not allow isolation of independent effect of autohemotherapy; full article not available

Zheng [16] Incomplete or internally inconsistent results in abstract; full article not available

Ou [17] Incomplete or internally inconsistent results in abstract; full article not available

Xiang [46] Insufficient detail in abstract to compute effect size; full article not available

Xiao [47] No abstract or full article available

Yang [48] Incomplete or internally inconsistent results in abstract; full article not available

You [49] No results related to research questions; full article not available

Patil [50] No results related to research questions

Khan [51] No empirical results reported in letter

Fang [52] Design does not allow isolation of independent effect of autohemotherapy; full article not available

Yu [18] Incomplete or internal inconsistent results in abstract; full article not available

Jeon [53] No results related to research questions

Sheikhi [54] Insufficient detail in letter to compute effect size or describe design; no further information available

Nahm [55] Treatment consisted of autologous immunoglobulin, not whole blood or serum

Cho [56] Treatment consisted of two variants of autologous plasma, no results related to research questions

\section{TABLE 1: Studies excluded from the systematic review}

${ }^{1}$ First author's surname

Patient Characteristics, Treatment Procedures, and Study Methods

Table 2 shows some of the key characteristics of the eight included studies, which were published in journals since 2006. The studies were conducted in Europe and Asia. Only one study focused on atopic dermatitis [19]. The urticaria studies included patients with chronic spontaneous/idiopathic urticaria. Patients with physical urticarias (such as dermatographism, 


\section{Cureus}

cold urticaria, or pressure urticaria) were excluded from all urticaria studies, except Debbarman and colleagues' trial [20]. All studies excluded patients who were pregnant, receiving immunosuppressant treatment, or had serious medical conditions, and most studies excluded lactating women and persons receiving anticoagulant therapy. 


\section{Cureus}

\begin{tabular}{|c|c|c|c|c|c|c|c|}
\hline Study $^{1}$ & Year $^{2}$ & Place & Design & Sample Size & $\begin{array}{l}\text { Length of } \\
\text { Treatment }\end{array}$ & $\begin{array}{l}\text { Length } \\
\text { of } \\
\text { Follow- } \\
\text { up }\end{array}$ & Threats to Validity ${ }^{3}$ \\
\hline $\begin{array}{l}\text { Staubach } \\
\text { [5] }\end{array}$ & $\begin{array}{l}\leq \\
2006\end{array}$ & $\begin{array}{l}\text { Mainz, } \\
\text { Germany }\end{array}$ & $\begin{array}{l}\text { RCT: AWB } \\
\text { vs. placebo }\end{array}$ & $\begin{array}{l}48 \text { (23 AWB, } 25 \\
\text { placebo; } 29 \\
\text { ASST+, } 19 \\
\text { ASST-) }\end{array}$ & 8 weeks & $\begin{array}{l}4 \\
\text { weeks }\end{array}$ & $\begin{array}{l}\text { Investigators not } \\
\text { blinded; allocation not } \\
\text { concealed }\end{array}$ \\
\hline $\begin{array}{l}\text { Kocatürk } \\
\text { [21] }\end{array}$ & 2009 & $\begin{array}{l}\text { Istanbul, } \\
\text { Turkey }\end{array}$ & $\begin{array}{l}\text { RCT: AWB } \\
\text { vs. AS vs. } \\
\text { placebo }\end{array}$ & $\begin{array}{l}88 \text { (29 AWB, } 30 \\
\text { AS, } 29 \text { placebo; } \\
59 \text { ASST+, } 29 \\
\text { ASST-) }\end{array}$ & 10 weeks & $\begin{array}{l}4 \\
\text { weeks }\end{array}$ & $\begin{array}{l}\text { Investigators not } \\
\text { blinded; allocation not } \\
\text { concealed }\end{array}$ \\
\hline $\begin{array}{l}\text { Debbarman } \\
\text { [20] }\end{array}$ & $\begin{array}{l}2011- \\
2\end{array}$ & $\begin{array}{l}\text { Kolkata, } \\
\text { India }\end{array}$ & $\begin{array}{l}\text { RCT: AS vS. } \\
\text { placebo }\end{array}$ & $\begin{array}{l}111 \text { (54 AS, } 57 \\
\text { placebo; } 45 \\
\text { ASST+, } 66 \\
\text { ASST-) }\end{array}$ & 9 weeks & $\begin{array}{l}16 \\
\text { weeks }\end{array}$ & $\begin{array}{l}\text { Allocation not } \\
\text { concealed; sample } \\
\text { includes patients with } \\
\text { physical urticarias }\end{array}$ \\
\hline Chen [22] & $\begin{array}{l}2010- \\
1\end{array}$ & $\begin{array}{l}\text { Chongqing, } \\
\text { China }\end{array}$ & $\begin{array}{l}\text { RCT: AWB } \\
\text { vs. no } \\
\text { placebo } \\
\text { control }\end{array}$ & $\begin{array}{l}100 \text { (60 AWB, } 40 \\
\text { control; all } \\
\text { ASST+) }\end{array}$ & 12 weeks & $\begin{array}{l}12 \\
\text { weeks }\end{array}$ & $\begin{array}{l}\text { Investigators and } \\
\text { patients not blinded; } \\
\text { allocation not } \\
\text { concealed; lack of } \\
\text { placebo control }\end{array}$ \\
\hline Pittler [19] & $\begin{array}{l}\leq \\
2003\end{array}$ & Exeter, UK & $\begin{array}{l}\text { RCT: AWB } \\
\text { vs. placebo }\end{array}$ & $\begin{array}{l}30 \text { (15 AWB, } 15 \\
\text { placebo) }\end{array}$ & 5 weeks & $\begin{array}{l}5 \\
\text { weeks }\end{array}$ & None apparent \\
\hline $\begin{array}{l}\text { Abdallah } \\
\text { [23] }\end{array}$ & $\begin{array}{l}\leq \\
2012\end{array}$ & $\begin{array}{l}\text { Cairo, } \\
\text { Egypt }\end{array}$ & $\begin{array}{l}\text { RCT: AWB } \\
\text { vs. AS }\end{array}$ & $\begin{array}{l}30 \text { (15 AWB, } 15 \\
\text { AS; all ASST+) }\end{array}$ & 8 weeks & $\begin{array}{l}4 \\
\text { weeks }\end{array}$ & $\begin{array}{l}\text { Allocation not } \\
\text { concealed; no } \\
\text { information on blinding }\end{array}$ \\
\hline Tseng [25] & $\begin{array}{l}2006- \\
8\end{array}$ & $\begin{array}{l}\text { Taipei, } \\
\text { Taiwan }\end{array}$ & $\begin{array}{l}\text { Prospective } \\
\text { cohort } \\
\text { receiving } \\
\text { AWB }\end{array}$ & $\begin{array}{l}23 \text { (15 ASST+, } 8 \\
\text { ASST-) }\end{array}$ & 8 weeks & $\begin{array}{l}4 \\
\text { weeks }\end{array}$ & $\begin{array}{l}\text { Possible selection } \\
\text { biases in which patients } \\
\text { received AWB }\end{array}$ \\
\hline Bajaj [24] & $\begin{array}{l}\leq \\
2012\end{array}$ & $\begin{array}{l}\text { Allahabad, } \\
\text { India } 4\end{array}$ & $\begin{array}{l}\text { Prospective } \\
\text { cohort } \\
\text { receiving } \\
\text { AS }\end{array}$ & $\begin{array}{l}75 \text { (62 ASST+, } 13 \\
\text { ASST-) }\end{array}$ & 9 weeks & $\begin{array}{l}\text { 12-16 } \\
\text { weeks }\end{array}$ & $\begin{array}{l}\text { Selection bias in } \\
\text { patients included in } \\
\text { analysis (greater } \\
\text { attrition among ASST-) }\end{array}$ \\
\hline
\end{tabular}

\section{TABLE 2: Studies included in the systematic review}

Note: All studies focused on urticaria except Pittler, which focused on dermatitis. RCT = randomized controlled trial, AWB = autologous whole blood injections, AS = autologous serum injections, ASST = autologous serum skin test. 1: First author's surname. 2: Year study conducted. 3: Judgment based on information reported or not reported in article. 4: Inferred from author location. 
(39\%) in only one [20]. Patients tended to be young adults or middle-aged (mean age ranged from 25 to 43 years across studies).

Autohemotherapy and placebo treatments were administered once weekly for 5 to 12 weeks, and follow-up assessments were done four to 16 weeks after treatment ended (Table 1).

Clinicians drew blood from each patient (including placebo control patients) and then injected autologous whole blood, autologous serum, or saline solution intramuscularly into the patient's upper arm, thigh, or buttock. In the urticaria studies, the first injection dose was 2 or $2.5 \mathrm{ml}$. Subsequent doses for autologous whole blood ranged from 3 to $10 \mathrm{ml}$ and for autologous serum ranged from 2 to $5 \mathrm{ml}$. Placebo patients received the same doses of saline solution as autohemotherapy patients received of blood or serum. Pittler and colleagues [19] administered doses of 1, 2, 3, 2, and $1 \mathrm{ml}$ in the five weeks of treatment, respectively, for atopic dermatitis. During the course of all urticaria studies, patients were allowed to use antihistamines as rescue medications.

In the included urticaria studies, measures of symptom severity typically focused on patients' self-reported number, size, and duration of wheals (and sometimes also angioedema and erythema), intensity of itchiness, and antihistamine use. In Pittler, et al.'s atopic dermatitis trial, the measure of symptom severity was based on an observer's assessment of erythema, exudation, excoriation, dryness, cracking, and lichenification at six body sites (head and neck, trunk, arms, hands, legs, and feet).

The included studies have both methodological strengths and weaknesses. Pittler, et al. [19] addressed all major threats to validity. Staubach, et al. [5] and Kocaturk, et al. [21] stratified randomization by ASST status, and Staubach, et al. [5] and Pittler, et al. [19] also randomized patients within blocks of successive patients. Debbarman, et al. [20] blinded both patients and evaluators to treatment condition, Staubach, et al. [5] and Kocaturk, et al. [21] blinded only patients, Chen, et al. [22] blinded neither, and Abdallah, et al. [23] reported no information on blinding. All randomized trial teams conducted intent to treat analyses on patients who returned after the first dose and thus had at least some outcome data (Kocaturk, et al. [21] included only those who returned for at least the third dose). However, in reports of the urticaria randomized trials, there was no information about how random allocation to treatment arms was concealed prior to enrolling patients (Table 2).

Authors of three studies reported funding for their work - two from private foundations [5, 19] (including one focused on alternative medicine [5]) and one from a pharmaceutical company [24]. Apart from these funding sources, I found no other apparent conflicts of interest. All study teams were led by conventional dermatologists, and most were affiliated with university medical schools. All study teams were independent of each other, with no authors in common among the included reports. Only one study [22] was pre-registered. Three teams [5, 23, 25] included the raw data for the key outcomes in numeric or graphic form in their reports. No other authors mentioned that study data were available or archived publicly.

\section{Adverse Effects}

In most studies, authors noted that some to many patients reported short-lived $(<24 \mathrm{hr})$ soreness and/or bruising at the injection site. Chen, et al. [22] and Abdallah, et al. [23] found that such effects were more common in patients receiving larger doses. There appeared to be no differences in adverse effects between autohemotherapy and placebo saline injections. Most authors did not report such comparisons, and when reported, only one included quantitative results. Other adverse effects, when mentioned by authors, were rare and minor. 
Patients receiving autohemotherapy tended to report less severe urticaria and eczema symptoms at the end of follow-up than control patients (Figure 2). The three placebo-controlled trials for urticaria displayed substantial variation in the estimated effectiveness of autohemotherapy $\left(\mathrm{I}^{2}=.66\right)$. The weighted mean response ratio, $\mathrm{R}$, for these trials is 0.81 (95\% CI 0.53 - 1.23), indicating that the severity of autohemotherapy patients' symptoms at the end of follow-up was $19 \%$ lower on average than the severity of placebo patients' symptoms. The estimated effectiveness of autohemotherapy is greater in Chen, et al.'s [22] urticaria trial (involving ASST+ patients only, without placebo control; $\mathrm{R}=0.26,95 \% \mathrm{CI} 0.16-0.42$ ) and Pittler, et al.'s [19] placebo-controlled eczema trial ( $\mathrm{R}=0.46$, 95\% CI $0.24-0.89)$.

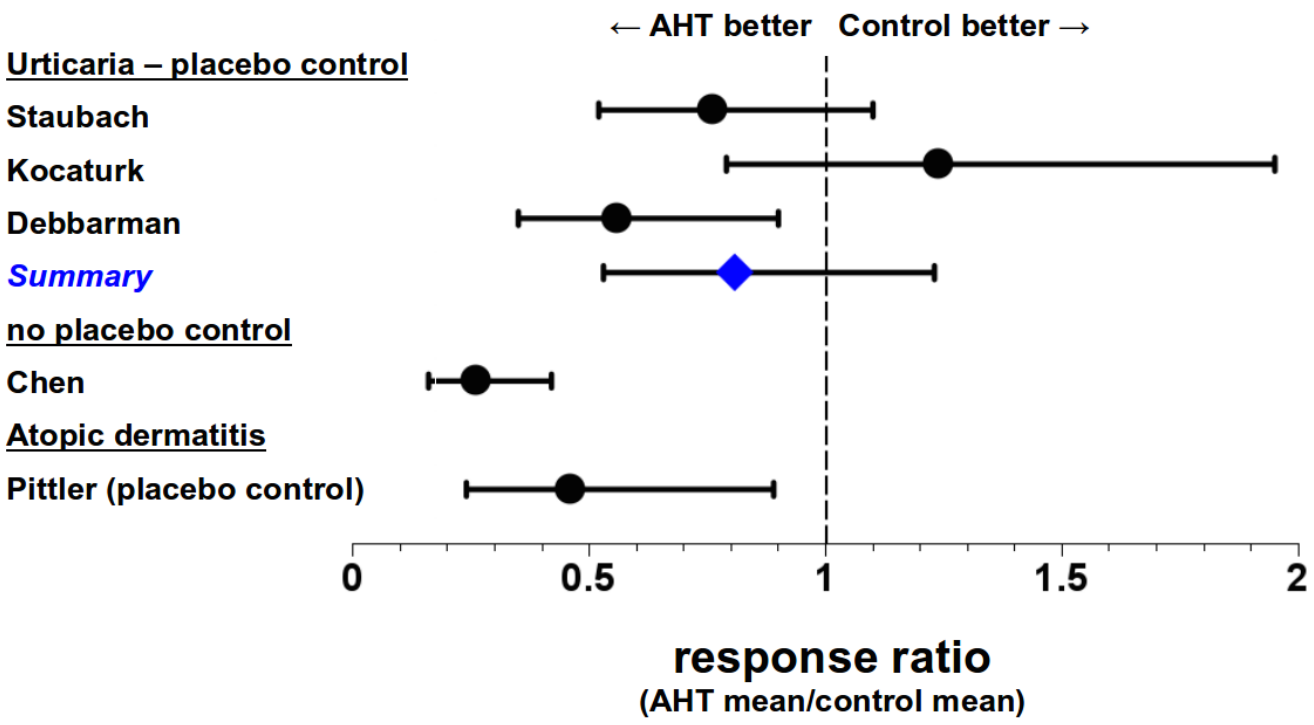

FIGURE 2: Effectiveness of autohemotherapy (AHT) compared to control on urticaria/eczema symptoms at end of follow-up (1-4 months after end of treatment)

Bars represent $95 \%$ confidence intervals.

In each of the urticaria trials, control patients reported moderate to large declines in symptom severity from baseline to the end of follow-up, on average (14\% for Staubach, et al. [5], 58\% for Kocaturk, et al. [21], 69\% for Debbarman, et al. [20], and 50\% for Chen, et al. [22]). In Pittler, et al.'s [19] eczema trial, however, the severity of placebo patients' symptoms increased by $7 \%$ on average from baseline to the end of follow-up.

Effectiveness of Autohemotherapy by ASST Status

Among ASST+ patients in the placebo-controlled urticaria trials, autohemotherapy reduced symptom severity by $23 \%$ on average relative to placebo (weighted mean $\mathrm{R}=0.77,95 \% \mathrm{CI}=0.44$ - 1.36; Figure 3). Similarly, among ASST- patients, autohemotherapy decreased severity of symptoms by $22 \%$ on average compared to placebo (weighted mean $\mathrm{R}=0.78,95 \%$ CI 0.59 1.04). In two of these trials (Staubach, et al. and Debbarman, et al. [5,20]), the relative effectiveness of autohemotherapy was greater (i.e., lower response ratio) in ASST+ patients than ASST- patients. In the other trial (Kocaturk, et al. [21]), though, ASST+ placebo patients improved more than those receiving autohemotherapy $(\mathrm{R}=1.47$, 95\% CI $0.76-2.87)$. 
Consequently, the heterogeneity of estimated effectiveness was much greater for ASST+ patients $\left(\mathrm{I}^{2}=.65\right)$ than ASST- patients $\left(\mathrm{I}^{2}=.00\right)$. Kocaturk, et al.'s [21] ASST+ placebo patients reported a dramatic improvement in symptom severity from the end of treatment (median urticaria activity score of 12 out of maximum possible 33) to the end of follow-up (median score of 0 ). No other patient group in the trial had such a large decline; indeed, ASST+ placebo patients had the lowest urticaria activity score of all patient groups at the end of follow-up. At the end of treatment in this trial, autohemotherapy had reduced symptoms $22 \%$ for ASST+ patients compared to placebo $(\mathrm{R}=0.78,95 \% \mathrm{CI} 0.55-1.11)$. When this result is combined with the only other placebo-controlled urticaria trial with end of treatment results for ASST+ patients [20], the weighted mean response ratio is 0.72 (95\% CI $0.64-0.81$ ).
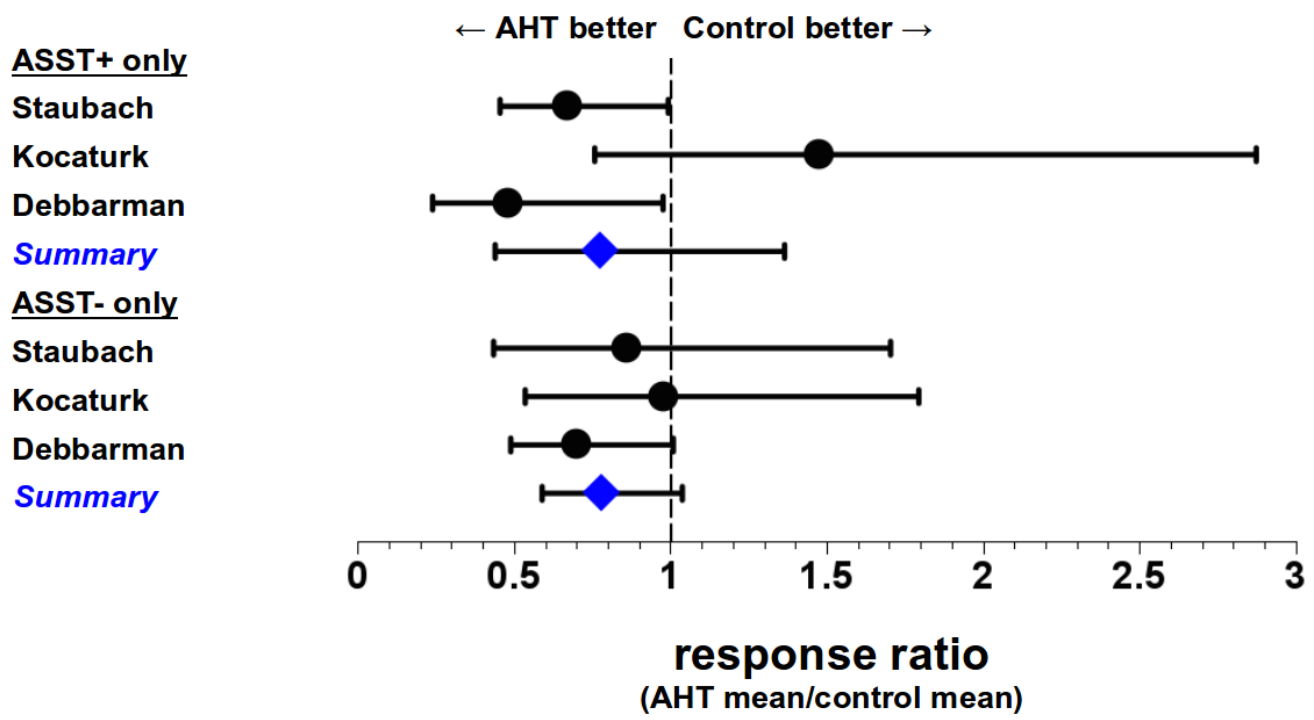

FIGURE 3: Effectiveness of autohemotherapy (AHT) compared to placebo control on urticaria symptoms at end of follow-up (1-4 months after end of treatment), by autologous serum skin test (ASST) status.

Bars represent $95 \%$ confidence intervals.

Direct comparisons between ASST+ and ASST- patients show consistent differences in their response to autohemotherapy (Figure 4). ASST+ patients who received autohemotherapy had $19 \%$ less severe urticaria symptoms at the end of follow-up than ASST- patients who received autohemotherapy, on average (weighted mean $\mathrm{R}=0.81$, 95\% CI $0.59-1.10$ ), despite having $5 \%$ greater severity of symptoms at baseline, on average (weighted mean R = 1.05, 95\% CI 1.00 1.11). The proportional change in symptom severity from baseline to the end of follow-up was calculable for individual patients who received autohemotherapy in two studies [5, 25]. In these studies, the reduction in symptom severity was considerably larger for ASST+ patients than for ASST- patients (weighted mean $\mathrm{R}=0.65$, 95\% CI $0.47-0.91$ ). (Bajaj, et al. [24] did not report enough information to compute an effect size for the effectiveness of autohemotherapy at the end of follow-up; the response ratio contrasting ASST+ and ASST- patients at the end of treatment was 0.65 (95\% CI $0.50-0.85))$. 


\section{Cureus}

End of follow-up scores

Debbarman

Kocaturk

Tseng

Summary

Prop. change to follow-up

Staubach

Tseng

Summary
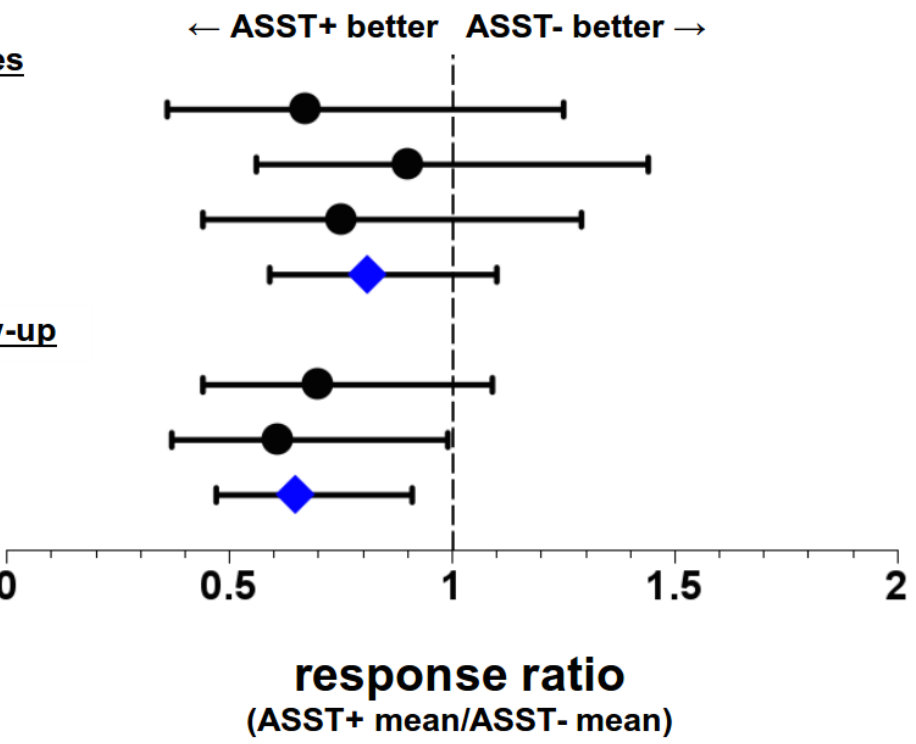

FIGURE 4: Comparative response to autohemotherapy at end of follow-up (1-4 months after end of treatment) for ASST+ and ASST- urticaria patients.

Bars represent $95 \%$ confidence intervals.

Comparative Effectiveness of Autologous Whole Blood Injections and Autologous Serum Injections

In two studies, there were no large or consistent differences in the effectiveness of autologous whole blood and autologous serum injections in reducing severity of urticaria symptoms (Figure 5). For ASST+ patients, the weighted mean response ratio is 0.88 (95\% CI 0.59-1.32), with no genuine variation in the two estimates $\left(\mathrm{I}^{2}=.00\right)$.

\section{ASST+ only \\ Kocaturk \\ Abdallah \\ Summary \\ ASST- only \\ Kocaturk}

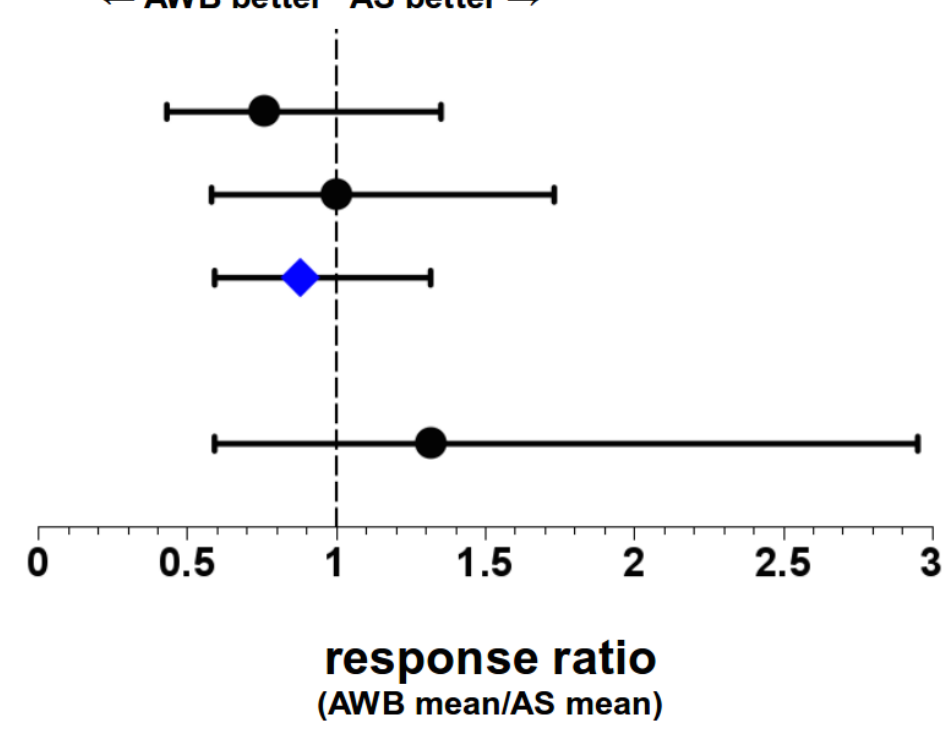


FIGURE 5: Comparative effectiveness of autologous whole blood injections (AWB) and autologous serum injections (AS) at end of follow-up (1-3 months after end of treatment) for ASST+ and ASST- urticaria patients

Bars represent $95 \%$ confidence intervals.

Effectiveness of Autohemotherapy in Relation to Symptom Severity at Baseline

The severity of symptoms at baseline was not consistently related to patients' response to autohemotherapy for urticaria (Figure 6). There were no obvious nonlinear associations present in any study. For both ASST+ and ASST- patients, the correlations between baseline symptoms and the proportional change in symptom severity from baseline to the end of follow-up varied in direction across studies. However, the genuine variation in these correlations was low to moderate (ASST $+\mathrm{I}^{2}=.39$, ASST- $\mathrm{I}^{2}=.00$ ) because the correlations are based on very small subsamples and thus have high sampling errors. The weighted mean correlation for ASST+ patients is .26 (95\% CI -.12 - .58) and for ASST- patients is -.11 (95\% CI -.62 - .47). Positive correlations indicate that patients with more severe symptoms at baseline respond less to autohemotherapy than patients with less severe symptoms at baseline.

ASST+ only
Staubach
Abdallah
Tseng
Summary
ASST- only
Staubach
Tseng
Summary

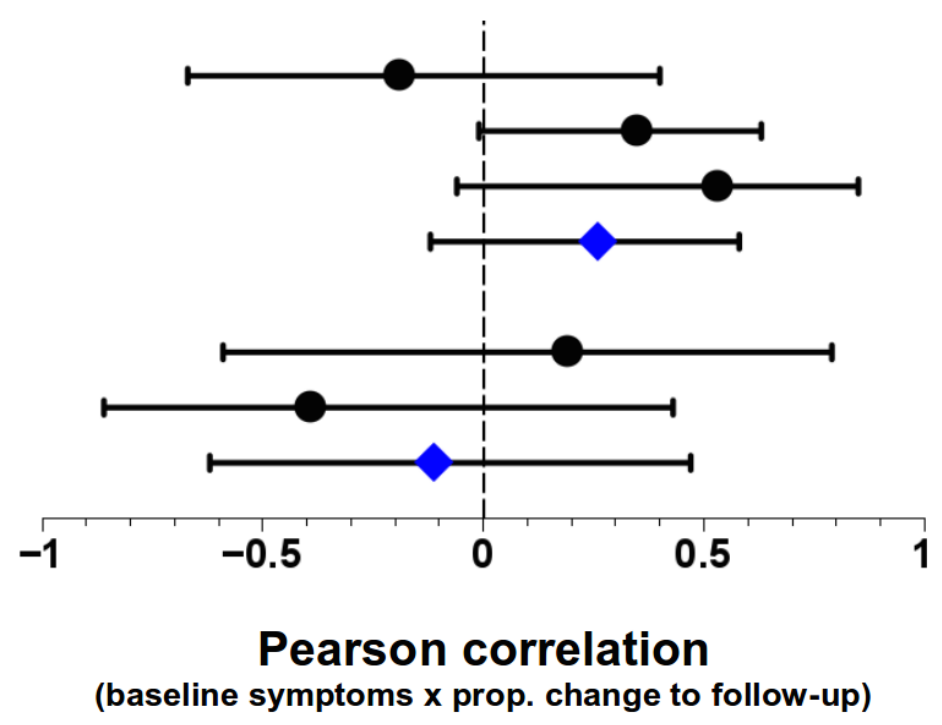

FIGURE 6: Pearson correlations between baseline symptoms and proportional change in symptom severity from baseline to end of follow-up for ASST+ and ASST- urticaria patients.

Bars represent $95 \%$ confidence intervals.

Response to Placebo Injections by ASST Status

ASST+ urticaria patients responded slightly and consistently more to placebo injections than 
did ASST- urticaria patients (Figure 7). ASST+ placebo patients reported less severe symptoms at the end of follow-up than ASST- placebo patients (weighted mean R $=0.88,95 \%$ CI 0.63 $\left.1.22, \mathrm{I}^{2}=.00\right)$, even though ASST+ patients had more severe symptoms at baseline than ASSTpatients (weighted mean $\mathrm{R}=1.03,95 \% \mathrm{CI}=1.01-1.05, \mathrm{I}^{2}=.00$ ). ASST+ patients reported a larger proportional improvement in symptoms from baseline to the end of follow-up than ASST- patients in Staubach, et al.'s [5] trial $(\mathrm{R}=0.89,95 \%$ CI $0.52-1.52)$.

End of follow-up scores

Debbarman

Kocaturk

Summary

Prop. change to follow-up

Staubach

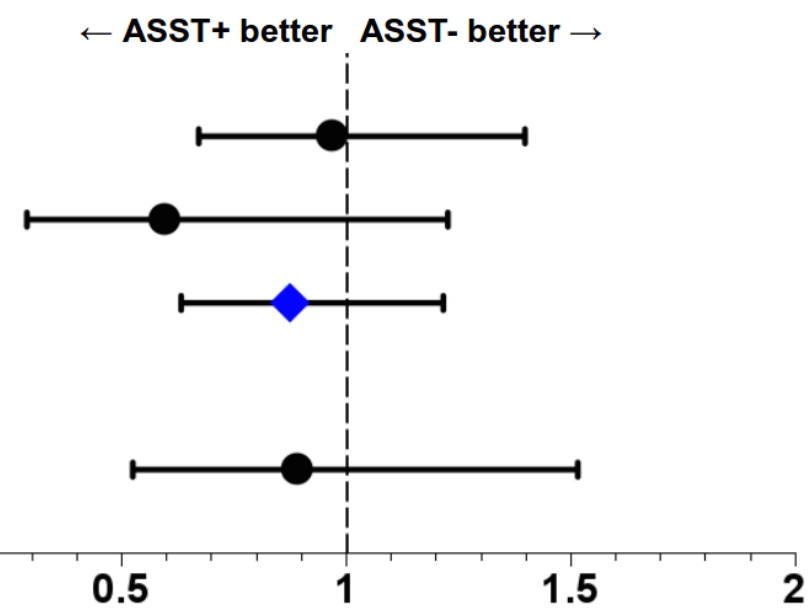

response ratio

(ASST+ mean/ASST- mean)

FIGURE 7: Comparative response to placebo saline injections at end of follow-up (1-4 months after end of treatment) for ASST+ and ASST- urticaria patients

Bars represent $95 \%$ confidence intervals.

Other Aspects of Results

There are too few studies with comparable designs and outcome measures to conduct any of the planned subgroup analyses beyond the comparisons by ASST status. Nonetheless, several observations are relevant. The two randomized trials for urticaria with the largest samples [20, 22] also had the largest estimated effects of autohemotherapy, and the very well-designed trial for eczema [19] had the largest estimated effect for a placebo-controlled trial. One of the urticaria trials [22] was pre-registered, but included controls without placebo. The placebocontrolled urticaria trial with the largest estimated effect [20] also was the most recently published. There was no obvious association between treatment duration (Table 1) and estimated effectiveness (Figure 1) and (Figure 2). In the four placebo-controlled trials for urticaria and eczema, three had post-treatment follow-up periods of four to five weeks [5, 19, 21] and one had a follow-up period of 16 weeks [20]. In the latter trial, symptom severity was virtually unchanged between four and 16 weeks post-treatment for ASST+ patients.

\section{Discussion}

Several decades ago, autohemotherapy was largely abandoned by conventional dermatologists for lack of supporting evidence, even though there had been no attempts to evaluate its effectiveness. In the last decade, several teams began building knowledge of this treatment for 
urticaria and eczema with a few systematic and rigorous studies. My systematic review of the literature shows that autohemotherapy does not have major side effects, and that minor adverse effects, such as soreness or bruising at the injection site, are short-lived. Overall, autohemotherapy tends to be somewhat more effective than control therapy across studies, although the advantage is not statistically reliable. ASST+ urticaria patients display a moderately better response to autohemotherapy than ASST- patients. Based on the limited evidence available, autologous whole blood and autologous serum injections appear to have similar effectiveness. Furthermore, the severity of symptoms prior to treatment is not consistently related to patients' apparent response to autohemotherapy.

More randomized trials are required to narrow the range of uncertainty in estimated effectiveness and provide clear guidance for treating urticaria and eczema patients generally. Nonetheless, the available evidence indicates autohemotherapy's effects are stronger for ASST+ than ASST- urticaria patients. Based on this result, I believe autohemotherapy is worth trying for ASST+ patients, especially before considering medications, beyond antihistamines, that have less evidence of effectiveness, greater cost, and higher risk of major adverse effects [2627].

Autologous whole blood injections and autologous serum injections are not decisively different in terms of their effectiveness for treating urticaria. Autologous serum injections require more time, equipment, expertise, and expense than autologous whole blood injections. Serum also is the majority constituent of whole blood. Indeed, in the included studies, autologous serum volumes injected tended to be about half as large as those for autologous whole blood, so the net amount of autologous serum injected was likely similar for both types of autohemotherapy. Thus, autologous whole blood injections have more advantages over autologous serum injection and no disadvantages, except for the larger dose of fluid injected and corresponding increased risk of injection site soreness and bruising.

Although autohemotherapy on average seems to reduce severity of symptoms relative to control therapy, symptoms can worsen for some patients who receive autohemotherapy. For example, $0-15 \%$ (median $=8 \%$ ) of ASST+ patients who received autohemotherapy had worse symptoms at the end of follow-up than at baseline [5, 23, 25]. However, 0-36\% (median = 20\%) of ASST+ urticaria patients [5, 21, 23-25] and 13\% of eczema patients [19] who received autohemotherapy apparently achieved complete remission (no symptoms) at the end of followup. Therefore, patients considering autohemotherapy should be informed about the range of likely outcomes.

If clinicians use a new sterile syringe and needle for each patient's blood draw and injection and follow all other procedures for safe injection, the risk of transmitting blood-borne pathogens during autohemotherapy is essentially eliminated. However, reused, contaminated equipment for modifying or treating autologous blood before reinjection can spread blood-borne infections efficiently, as shown in one large outbreak of Hepatitis B virus [28]. None of the studies included in my review modified autologous whole blood or serum in any way.

The mechanism underlying the apparent effectiveness of autohemotherapy is a matter of speculation, as there has been little systematic research on it. Several investigators hypothesized that autohemotherapy increases tolerance of circulating histamine-releasing factors by prompting a new immune response [5, 20-23, 29]. In this view, autohemotherapy is a kind of immunotherapy against self. A multi-center study is currently underway to elucidate possible mechanisms of effect for autohemotherapy (M. Maurer, personal communication, August 31, 2014).

Patients in the three trials who received placebo injections reported moderate to large 
decreases (14\% [5], 58\% [21], and 69\% [20]) in the severity of their urticaria symptoms from baseline to the end of follow-up. These improvements might represent regression to the mean, given that participating patients were recruited from clinics when they were presumably experiencing peak symptom severity. Natural variation in symptoms over time then might lead to improvements relative to when patients initially sought care, even if they had received no treatment. In contrast, Pittler and colleagues [19] recruited patients from the community. At enrollment, such patients would likely not have been experiencing peak symptom severity (for if they had been, they probably would have already sought care) and consequently would be less prone, as a group, to regress to the mean as they may have already been close to their average long-term level of symptoms. Placebo patients in this study did not show any improvement in their eczema from baseline to the the end of follow-up.

Chen and colleagues [22] did not give a placebo to their control patients recruited from a dermatology clinic in Chongqing, China, but they also reported a 50\% decline in severity of urticaria symptoms on average from baseline to the end of follow-up. Regression to the mean might account for this decline as well. Patients in both autohemotherapy and control arms did receive loratadine, however. If patients were not using antihistamines at study enrollment (as implied in the authors' report), then controls' improvement could also be due to the introduction of loratadine therapy.

Another possible reason for the dramatic declines in symptom severity for placebo patients is that placebo saline injections might function as a mild form of autohemotherapy. A placebo injection causes a little internal hemorrhaging and a small wound, as evidenced by reported bruising in many cases. Such an injury would generate a limited inflammatory and immune response to the patient's own blood (as well as the saline). This hypothesis is consistent with patient response to other kinds of placebos in randomized trials of urticaria treatments. For example, de Silva and colleagues [27] identified five randomized trials of montelukast in which control patients received oral placebos [30-34]. The changes in symptom severity from baseline to the end of treatment in these studies ranged from a $36 \%$ average decrease to a $5 \%$ average increase (median $=8 \%$ decrease). In five randomized trials of omalizumab [35-39], control patients received subcutaneous placebo injections. Placebo patients reported mean declines in symptom severity of $22 \%$ to $36 \%$ on average (median $=27 \%$ decrease) from baseline to mid- or end of treatment assessment. Patients in the three types of trials (autohemotherapy, montelukast, and omalizumab) were generally comparable in disease severity. Thus, placebo injections seem to induce greater improvements than oral placebos in urticaria patients. Intramuscular injections (as in the autohemotherapy trials) might provoke a greater inflammatory and immune response than subcutaneous injections (as in the omalizumab trials) and produce correspondingly greater reductions in symptom severity. The autohemotherapy trials also involved more injections at shorter intervals than in the omalizumab trials. Different types and frequencies of placebo injections may vary not only in their physiological effects but also in psychological impact.

In my systematic review, ASST+ urticaria patients consistently responded more favorably to placebo injections than ASST- urticaria patients did on average, although the difference is not statistically reliable. This observation is also consistent with the hypothesis that placebo injections are a mild version of autohemotherapy.

In light of these different observations, placebo injections might not be a neutral placebo therapy. Therefore, the placebo-controlled trials I reviewed may underestimate the effectiveness of autohemotherapy. Similarly, the omalizumab randomized trials with placebo injections for controls may also underestimate the effectiveness of omalizumab. Furthermore, bruises [19], punctures, and other wounds suffered occasionally might achieve the same beneficial effect as placebo injections, as they trigger new, small immune responses to the patient's own blood. 
Publication bias is present when the results available in the literature and summarized in a systematic review differ from results on the same topic that have not been published. Nearly every systematic review is at risk of publication bias, which represents perhaps the greatest threat to the cumulation of reliable scientific knowledge [39-40]. I sought both published and unpublished reports in any language, and there were no signs of potential publication bias in the limited number of studies I found. Nonetheless, publication bias still might have affected the results of my review.

My review has other limitations. All of the outcomes in the urticaria studies were based on selfreport. It would be useful to have complementary measures based on observer visual assessments of urticaria and angioedema, similar to the observer-based measures of atopic dermatitis used by Pittler, et al. [19]. Also, the psychometric properties of the symptom severity/activity measures have not been established beyond face validity. These composite scores are not technically ratio or even interval scale measures, even though they behave as ratio scale measures and can be interpreted and analyzed as such, as both the original authors and I did. It is unlikely that any psychometric problems with these measures could account for the results in my review. In addition, I estimated some results by hand from graphs in a few of the original reports. This undoubtedly leads to some error, but probably did not introduce bias to the review.

Further randomized controlled trials of autohemotherapy are necessary to clarify the effectiveness of this treatment for urticaria and eczema. Future trials could especially deepen understanding of autohemotherapy if they included arms with oral placebo and active oral medication in addition to autohemotherapy and placebo injection arms. The comparison between oral and injected placebos would help evaluate any potential benefits of placebo injections, and active oral medication (one that has been insufficiently evaluated) should be included for ethical purposes. Also, trials with longer treatment periods (and thus more injections) could indicate the optimal treatment duration, and trials with longer follow-up periods could reveal how long the apparent effects of autohemotherapy last. In addition, trials with autohemotherapy patients who have relapsed could assess the benefit of repeated cycles of autohemotherapy. Finally, to build trust and confidence in results, investigators should preregister their trials and publish or publicly archive de-identified study data.

\section{Conclusions}

Autohemotherapy is a safe treatment for urticaria and eczema. Across studies, autohemotherapy tends to be somewhat more effective overall than control therapy, but the advantage is not statistically reliable. Urticaria patients who test positive on the autologous serum skin test respond more favorably to autohemotherapy on average than those who test negative. More randomized trials are required to clarify the effectiveness of autohemotherapy.

\section{Additional Information \\ Disclosures}

Human subjects: All authors have confirmed that this study did not involve human participants or tissue. Animal subjects: All authors have confirmed that this study did not involve animal subjects or tissue. Conflicts of interest: In compliance with the ICMJE uniform disclosure form, all authors declare the following: Payment/services info: All authors have declared that no financial support was received from any organization for the submitted work. Financial relationships: All authors have declared that they have no financial relationships at present or within the previous three years with any organizations that might have an interest in the submitted work. Other relationships: All authors have declared that there are no other relationships or activities that could appear to have influenced the submitted work. 


\section{Acknowledgements}

I thank Dr. Emek Kocatürk for providing additional unpublished results. Stuart Brody, Barbara Leigh, John Potterat, two anonymous reviewers, and co-editor-in-chief, Alexander Muacevic, gave helpful comments on an earlier version of this article. There was no funding for my work described in this article.

\section{References}

1. Ravaut P: Essai sur L'Autohematotherapie dans quelques dermatoses (article in French) . Ann Dermatol Syphiligr . 1913, 4:292-6.

2. Spiethoff B: Therapeutischen Verwendung des Eigenserums (article in German) . Münchener Medizinische Wochenschrift. 1913, 60:521.

3. Gottheil WS, Satenstein DL: The autoserum treatment in dermatology. JAMA. 1914, 63:11901194. 10.1001/jama.1914.02570140044014

4. Mori O, Hashimoto T: Autologous whole blood intramuscular injection as a cure for chronic urticaria: Report of a patient in whom intradermal injection of autologous serum continued to cause a weal-and-flare response. Br J Dermatol. 1999, 140:1192.

5. Staubach P, Onnen K, Vonend A, Metz M, Siebenhaar F, Tschentscher I, Opper B, Magerl M, Lüdtke R, Kromminga A, Maurer M: Autologous whole blood injections to patients with chronic urticaria and a positive autologous serum skin test: A placebo-controlled trial. Dermatology. 2006, 212:150-9.

6. Pillsbury DM, Zimmerman MC, Baldridge GD: Experimental controls in clinical dermatologic investigation. J Invest Dermatol. 1950, 14:359-71.

7. Schäfer T: Epidemiology of complementary alternative medicine for asthma and allergy in Europe and Germany. Ann Allergy Asthma Immunol. 2004, 93:S5-10.

8. Sabroe RA, Grattan CE, Francis DM, Barr RM, Kobza Black A, Greaves MW: The autologous serum skin test: a screening test for autoantibodies in chronic idiopathic urticaria. Br J Dermatol. 1999, 140:446-52.

9. Konstantinou GN, Asero R, Maurer M, Sabroe RA, Schmid-Grendelmeier P, Grattan CE: EAACI/GA(2)LEN task force consensus report: the autologous serum skin test in urticaria . Allergy. 2009, 64:1256-68. 10.1111/j.1398-9995.2009.02132.x

10. Hedges LV, Gurevitch J, Curtis PS: The meta-analysis of response ratios in experimental ecology. Ecology. 1999, 80:1150-1156.

11. Borenstein M, Hedges LV, Higgins JPT, Rothstein HR: Introduction to Meta-Analysis. John Wiley \& Sons, Chichester; 2009.

12. DerSimonian R, Laird N: Meta-analysis in clinical trials. Control Clin Trials. 1986, 7:177-88.

13. Hedges LV, Vevea JL: Fixed and random effects models in meta-analysis . Psychological Methods. 1998, 3:486-504.

14. Higgins JPT, Thompson SG, Deeks JJ, Altman DG: Measuring inconsistency in meta-analyses . BMJ. 2003, 327:557-60.

15. Liang X, Deng B, Wang W: Observation on the effect of cetirizine combined with autohemotherapy on chronic urticaria (article in Chinese). China Trop Med. 2007, 6:954-955.

16. Zheng J, Wu X, Zheng J: Treatment of chronic urticaria: observation of therapeutic effect of autoblood point injection therapy combined with loratadine (article in Chinese). J Liaoning Univ Trad Chinese Med. 2008, 9:119-20.

17. Ou Q, Shen Y: Observation on the effect of clarityne combined with self-blood therapy on chronic urticaria (article in Chinese). Guangzhou Medical Journal. 2009, 5:24-25.

18. Yu F: Observation on the effect of reserpine, adrenosin with autohemotherapy on chronic urticaria (article in Chinese). Inner Mongolia J Trad Chinese Med. 2013, 24:48-49.

19. Pittler MH, Armstrong NC, Cox A, Collier PM, Hart A, Ernst E: Randomized, double-blind, placebo-controlled trial of autologous blood therapy for atopic dermatitis. Br J Dermatol. 2003, 148:307-13.

20. Debbarman P, Sil A, Datta PK, Bandyopadhyay D, Das NK: Autologous serum therapy in chronic urticaria: A promising complement to antihistamines. Indian J Dermatol. 2014, 59:375-82. 10.4103/0019-5154.135490

21. Kocatürk E, Aktaş S, Türkoğlu Z, Kavala M, Zindanci I, Koc M, Can B, Südoğan S: Autologous whole blood and autologous serum injections are equally effective as placebo injections in 
reducingdisease activity in patients with chronic spontaneous urticaria: A placebo controlled, randomized, single-blind study. J Dermatolog Treat. 2012, 23:465-71.

10.3109/09546634.2011.593485

22. Chen S, Zhai Z-F, Song Z, Feng L, Huang X, Zhou X, You Y, Zhong H, Wang H, Cheng L, Xiang M, Hao F: Autologous whole blood injections to patients with chronic spontaneous urticaria and positive autologous serum skin test: An efficacy evaluation (article in Chinese). Chinese J Dermatol. 2012, 45:470-473.

23. Abdallah MA, Elzamk M, Sallam MA: Autologous serum injection versus autologous blood in the treatment of chronic autoreactive urticaria. J Egyptian Women's Dermatologic Soc. 2012, 9:26-31.

24. Bajaj AK, Saraswat A, Upadhyay A, Damisetty R, Dhar S: Autologous serum therapy in chronic urticaria: Old wine in a new bottle. Indian J Dermatol Venereol Leprol. 2008, 74:109-13.

25. Tseng JT-P, Lee W-R, Lin S-S, Hsu C-H, Yang H-H, Wang K-H, Tsai H-H, Tsai T-H: Autologous serum skin test and autologous whole blood injections to patients with chronic urticaria: A retrospective analysis. Dermatol Sinica. 2009, 27:27-36.

26. Khan DA: Alternative agents in refractory chronic urticaria: evidence and considerations on their selection and use. J Allergy Clin Immunol Pract. 2013, 1:433-440.e1.

10.1016/j.jaip.2013.06.003

27. de Silva NL, Damayanthi H, Rajapakse AC, Rodrigo C, Rajapakse S: Leukotriene receptor antagonists for chronic urticaria: a systematic review. Allergy Asthma Clin Immunol. 2014, 10:24. 10.1186/1710-1492-10-24

28. Webster GJ, Hallett R, Whalley SA, Meltzer M, Balogun K, Brown D, Farrington CP, Sharma S, Hamilton G, Farrow SC, Ramsay ME, Teo C-G, Dusheiko GM: Molecular epidemiology of a large outbreak of hepatitis B linked to autohaemotherapy. Lancet. 2000, 356:379-84.

29. Burgess N: Further Observations on Autohæmotherapy. Br J Dermatol. 1933, 45:333-40.

30. Erbagci Z: The leukotriene receptor antagonist montelukast in the treatment of chronic idiopathic urticaria: A single-blind,placebo-controlled, crossover clinical study. J Allergy Clin Immunol. 2002, 110:484-8.

31. Reimers A, Pichler C, Helbling A, J. Pichler W, Yawalkar N: Zafirlukast has no beneficial effects in the treatment of chronic urticaria. Clin Exp Allergy. 2002, 32:1763-8.

32. Nettis E, Colanardi MC, Paradiso MT, Ferrannini A: Desloratadine in combination with montelukast in the treatment of chronic urticaria: A randomized, double-blind, placebocontrolled study. Clin Exp Allergy. 2004, 34:1401-7.

33. Kosnik M1, Subic T: Add-on montelukast in antihistamine-resistant chronic idiopathic urticaria. Respir Med. 2011, 105:S84-8. 10.1016/S0954-6111(11)70018-X

34. Wan KS: Efficacy of leukotriene receptor antagonist with an anti-H1 receptor antagonist for treatment of chronic idiopathicurticaria. J Dermatolog Treat. 2009, 20:194-7. 10.1080/09546630802607495

35. Maurer M, Altrichter S, Bieber T, Biedermann T, Bräutigam M, Seyfried S, Brehler R, Grabbe J, Hunzelmann N, Jakob T, Jung A, Kleine-Tebbe J, Mempel M,Meurer M, Reich K, Ruëff F, Schäkel K, Sengupta K, Sieder C, Simon JC, Wedi B, Zuberbier T, Mahler V, Staubach P: Efficacy and safety of omalizumab in patients with chronic urticaria who exhibit IgE against thyroperoxidase. J Allergy Clin Immunol. 2011, 128:202-209.e5. 10.1016/j.jaci.2011.04.038

36. Saini S, Rosen KE, Hsieh HJ, Wong DA, Conner E, Kaplan A, Spector S, Maurer M: A randomized, placebo-controlled, dose-ranging study of single-dose omalizumab in patients with H1-antihistamine-refractory chronic idiopathic urticaria. J Allergy Clin Immunol. 2011, 128:567-73.e1. 10.1016/j.jaci.2011.06.010

37. Kaplan A, Ledford D, Ashby M, Canvin J, Zazzali JL, Conner E, Veith J, Kamath N, Staubach P, Jakob T, Stirling RG, Kuna P, Berger W, Maurer M, Rosén K: Omalizumab in patients with symptomatic chronic idiopathic/spontaneous urticaria despite standardcombination therapy. J Allergy Clin Immunol. 2013, 132:101-9. 10.1016/j.jaci.2013.05.013

38. Maurer M, Rosén K, Hsieh H-J, Saini S, Grattan C, Gimenéz-Arnau A, Agarwal S, Doyle R, Canvin J, Kaplan A, Casale T: Omalizumab for the treatment of chronic idiopathic or spontaneous urticaria. NEJM. 2013, 368:924-35. 10.1056/NEJMoa1215372

39. Saini SS, Bindslev-Jensen C, Maurer M, Grob JJ, Bülbül Baskan E, Bradley MS, Canvin J, Rahmaoui A, Georgiou P, Alpan O, Spector S, Rosén K: Efficacy and Safety of Omalizumab in Patients with Chronic Idiopathic/Spontaneous Urticaria Who Remain Symptomatic on H1 Antihistamines: A Randomized, Placebo-Controlled Study. J Invest Dermatol. 2014, July 21, 


\section{4:10.1038/jid.2014.306}

40. Song F, Parekh S, Hooper L, Loke YK, Ryder J, Sutton AJ, Hing C, Kwok CS, Pang C, Harvey I: Dissemination and publication of research findings: an updated review of related biases . Health Technol Assess. 2010, 14:iii, ix-xi, 1-193. 10.3310/hta14080

41. Franco A, Malhotra N, Simonovits G: Social science. Publication bias in the social sciences: Unlocking the file drawer. Science. 2014, 345:1502-5. 10.1126/science.1255484

42. Hiraizumi T, Nakajima H, Kashima N, Kobayashi K, Ide H, Takahashi T: An application of autologous blood injection therapy in patients with chronic urticaria and its effects onthresholds of skin reaction (article in Japanese). Arerugi. 1988, 37:115-20.

43. Chopra A, Mamta, Chopra D: Autohaemotherapy in chronic urticaria . Indian J Dermatol Venereol Leprol. 1995, 61:323-4.

44. Yang D, Jiang Y, Zhang Y: A study on the efficacy and safety of auto-blood in the treatment of chronic idiopathic urticaria. Pract J Med Pharm. 2006, 1:38-40.

45. Min X, Yue Q: Autohemotherapy with combined TCM and Western medicine on chronic urticaria. World J Integrated Trad Western Med. 2008, 5:281-283.

46. Xiang Y, Ji Q, Cai H, Chen Y: Observation of clinical efficiency of compound glycyrrhizin combined with desloratadine and auto-blood therapy in the treatment of chronic urticaria (article in Chinese). Chinese J Dermatovenereol. 2010, 12:1173-1175.

47. Yang L: Clinical observation on the efficacy of self-blood therapy in the treatment of chronic urticaria (article in Chinese). Chinese Foreign Women Health. 2011, 6:47.

48. Xiao R: Effect of autologous transfusion therapy combined with loratadine (desloratidine) on chronic urticaria (article in Chinese). China Trop Med. 2010, 6:738.

49. You H-S, Mun J-H, Jwa S-W, Song M, Kim H-S, Ko H-C, Kim M-B, Kim B-S: Autologous whole blood injection for antihistamine resistant chronic spontaneous urticaria: A preliminary open-label trial. 64th Dermatology Conference, 2012, Korean Dermatological Association, Seoul. 2012, 64:300. http://www.papersearch.net/google_link/fulltext.asp? file_name=27728306.pdf..

50. Patil S, Sharma N, Godse K: Autologous serum therapy in chronic urticaria . Indian J Dermatol. 2013, 58:225-6. 10.4103/0019-5154.110833

51. Khan S: More robust evidence and safety checks are required before autologous serum therapy as treatment for chronic urticaria can be recommended. Indian J Dermatol. 2013, 58:316-7. 10.4103/0019-5154.113964

52. Fang L, Tan C, Peng J: Clinical observation on blood-nourishing and wind-expelling needling method plus autohemotherapy for chronic urticaria (article in Chinese). Shanghai J Acu-mox. 2013, 32:115-17. 10.3969/j

53. Jeon IK, On HR, Oh SH, Hann SK: Three cases of pruritic urticarial papules and plaques of pregnancy (PUPPP) treated with intramuscular injection of autologous whole blood. J Eur Acad Dermatol Venereol. 2014, [Epub ahead of print]. 10.1111/jdv.12414

54. Sheikhi A, Azarbeig M, Karimi H: Autohemotherapy in chronic urticaria: what could be the autoreactive factors and curative mechanisms?. Ann Dermatol. 2014, 26:526-7. 10.5021/ad.2014.26.4.526

55. Nahm D-H, Cho S-M, Kim M-E, Kim Y-J, Jeon S-Y: Autologous immunoglobulin therapy in patients with severe recalcitrant atopic dermatitis: A preliminary report. Allergy Asthma Immunol Res. 2014, 6:89-94. 10.4168/aair.2014.6.1.89

56. Cho S-M, Kim M-E, Kim J-Y, Park J-C, Nahm D-H: Clinical efficacy of autologous plasma therapy for atopic dermatitis. Dermatology. 2014, 228:71-7. 10.1159/000356387 